\title{
Comparison of Fat Harvested from Flank and Falciform Regions for Stem Cell Therapy in Dogs.
}

Jifcovici A, Solano M.A, Fitzpatrick N, Findji L, Blunn G, Sanghani-Kerai A.

\section{Introduction}

Adipose tissue has recently gained attention as a source of mesenchymal stem cells (AdMSCs), because it is easier to acquire in larger volumes, the harvest is less painful, there are lower surgical risks, and there is minimal morbidity to the patient. Adipose tissue can be collected from multiple sites, such as the inguinal, flank, omental, neck, scapular, falciform, and other regions. The harvested fat is broken down and centrifuged to obtain a pellet. The pellet contains the stromal vascular fraction (SVF), which is composed of a mixture of cells including AdMSCs, erythrocytes, pericytes, fibroblasts, endothelial cells, smooth muscle cells, leukocytes, mast cells, and pre-adipocytes.

The AIM of this study was to investigate AdMSCs from the falciform and flank regions by quantifying the stromal vascular fractions (SVFs), colony forming units (CFU), aspect ratio of the cells, as well as the days needed to count the $\mathrm{CFU}$. We also aimed to report surgical site complications after harvesting the fat from the two different anatomical regions and compare the quality of fat contingent on the age and weight of the of the patient. This could help in the determination of the minimum amount of fat required to obtain AdMSCs for treatment of degenerative joint disease.

\section{Materials and Methods}

With informed owner consent, fat tissue was harvested from either the flank (21 dogs) or falciform region (17 dogs) from separate dogs. The fat tissue was weighed, minced, enzymatically digested and centrifuged to obtain the SVF. The number of nucleated cells in the SVF was counted. The SVF was cultured in vitro and the cell growth was assessed by counting the CFU per gram of fat. The aspect ratio of the cells was measured by dividing the length and width of the cells using images obtained using a microscope.

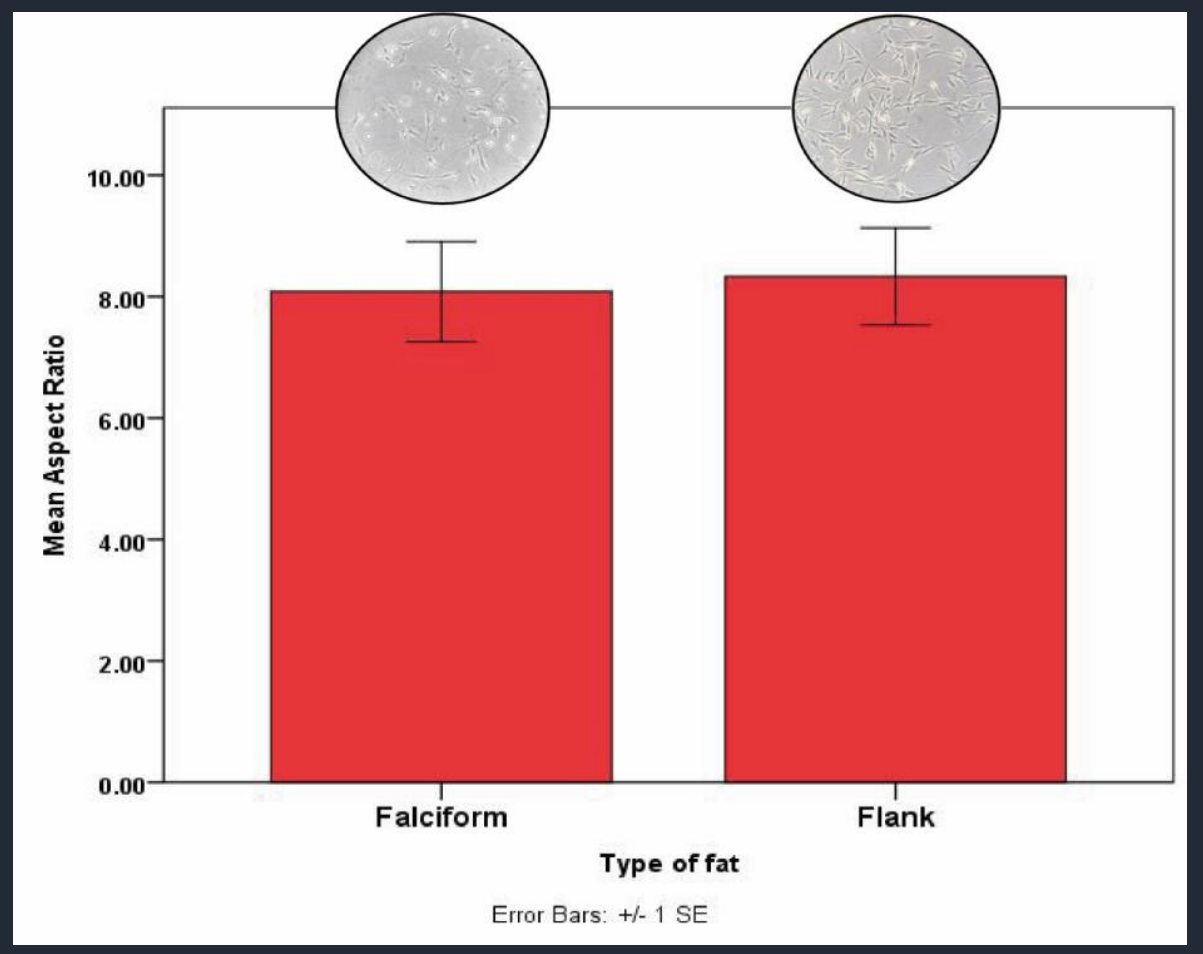

Fig. 1 The mean aspect ratio (ratio of length/width) of AdMSCs harvested from the flank and falciform region were very similar $(p=0.83)$. The error bars represent 1 standard error. Amplification factor of the image-5x.

\section{Results}

- No significant difference in the number of nucleated cells in the SVF from the two sites.

- The CFU/g of fat from falciform was $378.9+/-293 \mathrm{~g}$ and from flank was $486.8+/-517 \mathrm{~g}$, and this was also insignificant.

- Neither age nor weight of the patient had an impact on the SVF or $\mathrm{CFU} / \mathrm{g}$.

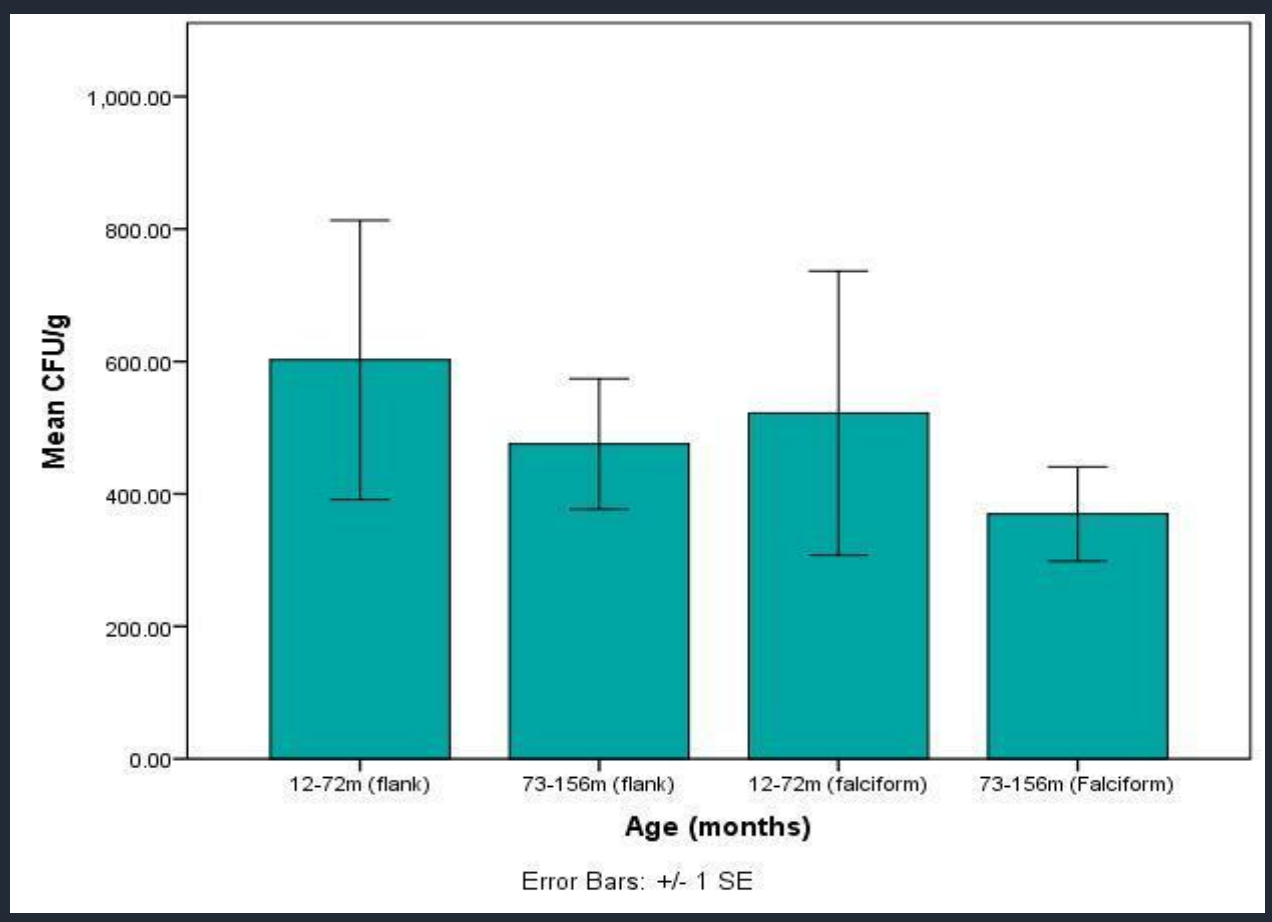

Fig. 2 The colony forming units (CFU)/g of fat in relationship to the age of the patient showing no correlation in either of the anatomical region.

\section{Discussion}

- Irrespective of the harvest site, the stem cell quantity, measured by the CFU counts, was not significantly different.

- There was no difference in cell morphology between the adipose tissue harvested from the two anatomical regions: flank and falciform.

- The ideal site for collecting adipose tissue from a clinical perspective would be the site that causes minimal morbidity, consistently has a large amount of fat for extraction, and yields a large SVF, which should be proportional to the fat obtained.

\section{Conclusion}

No surgical complications were reported from either of the sites. Harvesting fat for stem cell therapy for intra-articular therapy of degenerative joint disease can be an easy and fast process when obtaining the fat either from the flank or the falciform region, and it is not age or weight dependent. The harvest site for clinical canine patients can be left to the surgeon's discretion and comfort. 